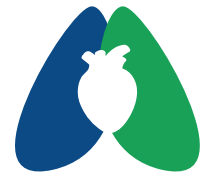

ASSOBRAFIR

C I Ê N C I A

\section{Desfechos clínicos de pacientes submetidos à ventilação mecânica invasiva em uma UTI neurocirúrgica ${ }^{a}$}

\author{
Clinical outcomes of patients undergoing invasive mechanical \\ ventilation in a neurosurgical ICU
}

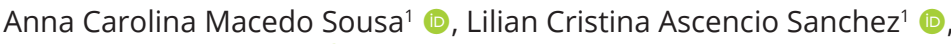 \\ Lucas Lima Ferreira $2,3 *$ (1)

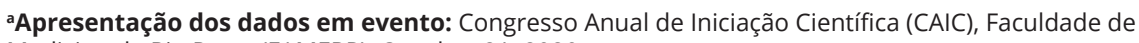 \\ Medicina de Rio Preto (FAMERP), Outubro 21, 2020. \\ 'Programa de Pós-graduação em Fisioterapia, União das Faculdades dos Grandes Lagos (UNILAGO), São José \\ do Rio Preto, SP, Brasil \\ 2Departamento de Pós-graduação em Fisioterapia, União das Faculdades dos Grandes Lagos (UNILAGO), São \\ José do Rio Preto, SP, Brasil \\ ${ }^{3}$ Hospital de Base, Fundação Faculdade Regional de Medicina de São José do Rio Preto (FUNFARME), São José \\ do Rio Preto, SP, Brasil
}

Como citar: Sousa ACM, Sanchez LCA, Ferreira LL. Desfechos clínicos de pacientes submetidos à ventilação mecânica invasiva em uma UTI neurocirúrgica. ASSOBRAFIR Ciênc. 2021;12:e42286. https://doi. org/10.47066/2177-9333.AC.2020.0021

\begin{abstract}
Resumo
Introdução: Pacientes com doenças neurológicas internados em unidade de terapia intensiva (UTI) sob ventilação mecânica (VM) apresentam maior chance de complicações respiratórias. Objetivo: Comparar os desfechos clínicos de pacientes submetidos à VM em uma UTI neurocirúrgica. Métodos: Estudo retrospectivo, realizado na UTI neurocirúrgica de um hospital escola. Foram coletados: idade, sexo, doença que o levou a internação, presença de comorbidades, valor do Simplified acute physiology score (SAPS III), tempo de permanência em VM, sucesso ou falha da extubação, tempo de permanência na UTI e desfechos na unidade como alta ou óbito. Os pacientes foram divididos em grupo sucesso (GS) e grupo falha (GF) do desmame. Resultados: Foram incluídos 158 pacientes, 75\% no GS e $25 \%$ no GF, com predomínio do sexo masculino em ambos os grupos. O diagnóstico mais prevalente foi o traumatismo cranioencefálico no GS e a ressecção de tumor cerebral no GF. O GF apresentou escore SAPS III $(68,8 \pm 14,6$ versus $54,3 \pm 17,4 p=0,003)$ e permaneceu mediana de tempo significativamente maiores em internação na UTI (12,5 [5-35] versus 9 [2-49] dias $p=0,002)$ e em VM $(3,5$ [1-14] versus 2 [1-12] dias $p=0,05)$ que o GS. O GS apresentou como desfecho alta da UTI significativamente maior ( $90 \%$ versus $67,5 \% \mathrm{p}=0,001$ ) que o GF. Conclusão: Os pacientes que falharam no desmame ventilatório apresentam maior índice preditivo para mortalidade na UTI, maior tempo de VM e internação, menor taxa de alta e maior taxa de óbitos na comparação com os pacientes que evoluíram com sucesso no desmame ventilatório.
\end{abstract}

Palavras-chave: Respiração Artificial; Unidade de Terapia Intensiva; Desmame do Respirador; Fisioterapia.

\begin{abstract}
Background: Patients with neurological diseases admitted to the intensive care unit (ICU) under mechanical ventilation (MV) have a greater chance of respiratory complications. Aim: To compare the clinical outcomes of patients undergoing MV in a neurosurgical ICU. Methods: Retrospective documentary study, carried out in the neurosurgical ICU of a teaching hospital. Were collected: age, sex, pathology that led to hospitalization, presence of comorbidities, value of Simplified acute physiology score (SAPS III), length of stay on MV, success or failure of extubation, length of stay in the ICU and outcomes in the unit such as discharge or death. Patients were divided into the success group (SG) and the failure group (FG) of weaning. Results: 158 patients were included, 75\% in SG and 25\% in FG, with a male predominance in both groups. The most prevalent diagnosis was traumatic brain injury in the SG and resection of a brain tumor in the FG. The FG had a SAPS III score $(68.8 \pm 14.6$ versus $54.3 \pm 17.4 \mathrm{p}=0.003)$ and remained significantly longer in the ICU $(12.5$ [5-35] versus
\end{abstract}

Submissão em: Dezembro 10, 2020 Aceito em: Junho 08, 2021

Estudo realizado em: União das Faculdades dos Grandes Lagos, São José do Rio Preto, $\mathrm{SP}$, Brasil.

Aprovação ética: CAAE

31914620.8.0000.5489 da União das

Faculdades dos Grandes Lagos, n 4.062.514

*Autor correspondente: Lucas Lima Ferreira. E-mail: lucas_lim21@hotmail.com 
9 [2-49] days $p=0.002)$ and in MV (3.5 [1-14] versus 2 [1-12] days $p=0.05)$ than the SG. The SG presented a significantly higher ICU outcome ( $90 \%$ versus $67.5 \% \mathrm{p}=0.001)$ than the FG. Conclusion: Patients who failed ventilatory weaning have a higher predictive index for ICU mortality, longer MV and hospital stay, lower discharge rate and higher death rate compared to patients who have successfully evolved in ventilatory weaning.

Keywords: Artificial Respiration; Intensive Care Unit; Weaning from Respirator; Physical Therapy.

\section{INTRODUÇÃO}

A unidade de terapia intensiva (UTI) é considerada como área de atendimento e monitoramento ao paciente crítico que necessita de auxílio constante, permanente e especializado. Neste setor são utilizados recursos tecnológicos apropriados para a monitorização contínua dos sinais vitais do paciente, bem como a intervenção em caso de descompensação'.

Grande parte dos pacientes internados na UTI apresentam alterações respiratórias importantes, sendo incapazes de manter uma ventilação espontânea eficaz, necessitando, portanto, de suporte ventilatório ${ }^{2}$. O suporte ventilatório ou ventilação mecânica (VM) é definido como um recurso para auxílio no tratamento de pacientes com insuficiência respiratória aguda (IRpA) ou crônica agudizada ${ }^{3}$. Dentre estes destaca-se a ventilação mecânica invasiva (VMI) que consiste em um método invasivo por tubo endotraqueal ou pela traqueostomia, substituindo a respiração espontânea ${ }^{4}$.

De acordo com dados da literatura, $40 \%$ dos pacientes internados em UTI são submetidos a VM invasiva ${ }^{5}$, destes, $20 \%$ correspondem a distúrbios neurológicos agudos, sendo doença neuromuscular, disfunção do sistema nervoso central ou coma, as principais razões para introdução da VM ${ }^{6}$.

A retirada da respiração artificial é um processo complexo que ocupa em torno de $35 \%$ a $40 \%$ do período total de VM. O desmame ventilatório é definido como o processo de substituição da ventilação artificial para a espontânea nos pacientes que permaneceram em VMI por mais de 24 horas $^{7}$.

De acordo com o III Consenso Brasileiro de Ventilação Mecânica ${ }^{8}$, o processo de desmame pode ser longo e envolve diversas estratégias, entre elas, o teste de respiração espontânea (TRE). O TRE é aplicado para avaliar o sucesso no desmame e predizer o sucesso da extubação. Podendo ser realizado através da conexão de uma peça em forma de "T" no tubo endotraqueal, com uma fonte rica em oxigênio, ou através de pressão positiva contínua em vias aéreas (CPAP) de $5 \mathrm{cmH}_{2} \mathrm{O}$, ou $7 \mathrm{cmH}_{2} \mathrm{O}$ em ventilação com pressão de suporte (PSV). O paciente deve permanecer em TRE de 30-120 minutos, sendo monitorizados os sinais de possível falha. Considera-se sucesso, caso o paciente mantenha estabilidade hemodinâmica, padrão respiratório, troca gasosa e conforto adequados ${ }^{9}$.

O sucesso do desmame ventilatório tem sido definido como a manutenção da ventilação espontânea durante pelo menos 48 horas após a interrupção da ventilação artificial. Em contrapartida considera-se insucesso ou falha, se houver necessidade de retorno à ventilação artificial neste período. Quando aplicado de forma correta diminui índice de reintubações, infecções respiratórias e o tempo de permanência hospitalar, aumentando a sobrevida dos pacientes submetidos a $\mathrm{VM}^{7}$.

A VM prolongada é definida na literatura como superior a 24 horas, mais de 96 horas, mais de 7 dias, mais de 29 dias e necessidade de suporte com VM após alta da UTII0,11. O consenso de 2005 da Associação Nacional de Direção Médica dos Cuidados Respiratórios (NAMDRC) define a VM prolongada sendo ela por mais de 6 horas diárias e com duração de 21 dias consecutivos. A VM prolongada está diretamente associada com o aumento de mortalidade precoce e a longo prazo, pior qualidade de vida, maiores demandas e gastos com assistência médica ${ }^{12}$.

O uso prolongado da VM aumenta as chances de complicações pulmonares devido à diminuição da capacidade pulmonar e com isso, cresce os índices de mortalidade ${ }^{13}$. Além de estar associada ao desenvolvimento de patologias como pneumonia associada à ventilação (PAV), disfunção diafragmática induzida pela ventilação, lesão pulmonar induzida pela ventilação e polineuropatia ${ }^{14}$.

Segundo Gutiérrez et al. ${ }^{15}$ pacientes com doença neurológica submetidos a VM apresentam maior risco de $\mathrm{PAV}$, número maior de traqueostomias, mais tempo em VM e mortalidade ainda maior.

A hipótese do presente estudo é que pacientes neurológicos que evoluíram com sucesso no desmame da VM permaneceram menor tempo em VM, menor tempo de internação na UTI, apresentaram menores taxas de complicações e obtiveram maiores taxas de sobrevida e alta da unidade. Assim, o objetivo do presente estudo foi comparar os desfechos clínicos dos pacientes que evoluíram com sucesso ou falha no desmame da ventilação mecânica invasiva em uma UTI neurocirúrgica.

\section{MÉTODOS}

Trata-se de um estudo retrospectivo, realizado na Unidade de Terapia Intensiva Neurocirúrgica do $7^{\circ}$ andar do Hospital de Base da Fundação Faculdade Regional de Medicina (FUNFARME), na cidade de São José de Rio Preto - SP, Brasil.

Foram incluídos no estudo, pacientes com idade maior ou igual a 18 anos que estiveram sob VMI há mais de 
24 horas na UTI Neurocirúrgica, no período de junho de 2014 a abril de 2020.

Pacientes com doença neuromuscular conhecida, além daqueles com dados imprecisos sobre a duração da ventilação antes da admissão à UTI, ou que tivessem restrição ou suspensão de medidas terapêuticas durante a permanência na UTI foram excluídos do estudo.

Foram coletados dados como, idade, sexo, doença que o levou a internação, presença de comorbidades como hipertensão arterial, diabetes mellitus, etilismo, tabagismo, entre outras, valor do Simplified acute physiology score (SAPS III), tempo de permanência em VMI, sucesso ou falha da extubação, tempo de permanência em dias na UTI, e desfechos da unidade como alta ou óbito em formulário próprio estruturado pelos pesquisadores.

Na UTI neurocirúrgica do Hospital de Base utiliza-se um protocolo de desmame da VM (Figura 1), que considera critérios para extubação definidos pela coordenação do serviço de fisioterapia baseado em evidências da literatura e chancelado pela instituição. A falha da extubação é considerada quando há necessidade de retorno à VM invasiva nas primeiras 48 horas após retirada da via aérea artificial.
O sistema prognóstico SAPS III é composto de 20 diferentes variáveis facilmente mensuráveis na admissão do paciente a UTI. As variáveis são divididas em três partes, variáveis demográficas, razões pela admissão na UTI e variáveis fisiológicas, elas representam o grau de comprometimento da doença e avaliação do estado de saúde prévio à admissão hospitalar, indicadora da condição pré-mórbida. Para cada uma das variáveis analisadas confere-se um peso, conforme a gravidade do distúrbio fisiológico. Na teoria, o menor valor atribuído pelo escore é 16 e o maior é 217 pontos. As variáveis fisiológicas que compõem o escore fisiológico agudo são: temperatura, pressão arterial sistólica, frequência cardíaca e respiratória, oxigenação, pH arterial, sódio, potássio, creatinina, bilirrubina, hematócrito, leucócitos, plaquetas e escala de coma de Glasgow ${ }^{16,17}$.

Os dados foram coletados do livro de desmame ventilatório do Serviço de Fisioterapia e dos prontuários clínicos dos pacientes da UTI neurocirúrgica do Hospital de Base de São José de Rio Preto - SP, Brasil. A coleta de dados foi realizada nos meses de maio e junho de 2020.

O trabalho seguiu os princípios éticos estabelecidos na Resolução n. ${ }^{\circ}$ 466/2012 do Conselho Nacional em

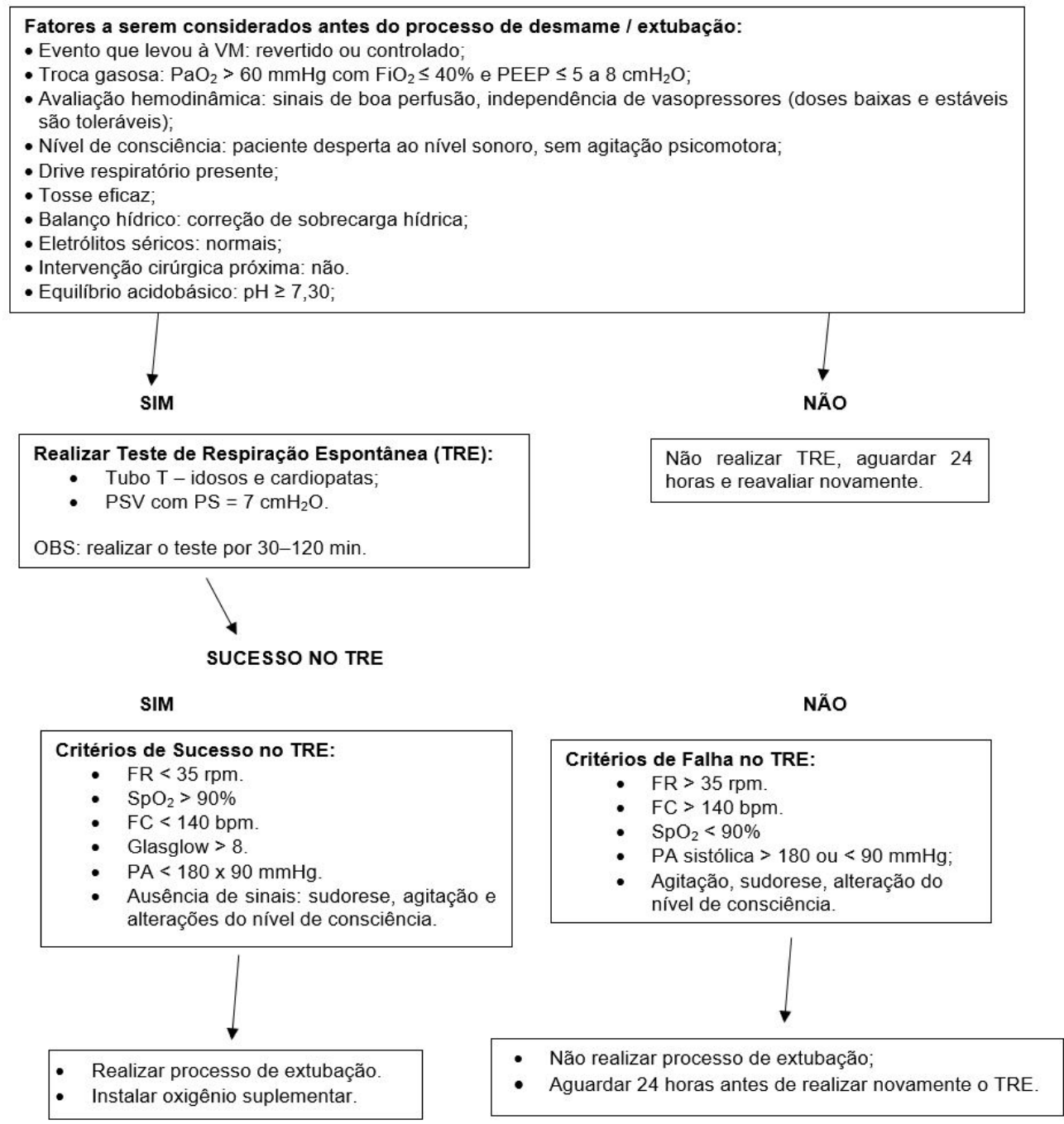

Figura 1. Protocolo institucional de desmame/extubação do Serviço de Fisioterapia. 
Saúde e foi submetido ao Comitê de Ética em Pesquisa da União das Faculdades dos Grandes Lagos (UNILAGO) pelo CAAE 31914620.8.0000.5489 e aprovado sob parecer n. ${ }^{\circ}$ 4.062.514. Foi autorizado pelo comitê de ética, dispensa do Termo de Consentimento Livre e Esclarecido por se tratar de um estudo documental que utilizou dados secundários.

A análise estatística foi realizada de forma descritiva quantitativa na qual variáveis contínuas foram apresentadas em forma de média \pm desvio-padrão ou mediana (variação interquartil IQR) e as variáveis categóricas foram descritas em porcentagens e frequências. Além disso, foi analisada

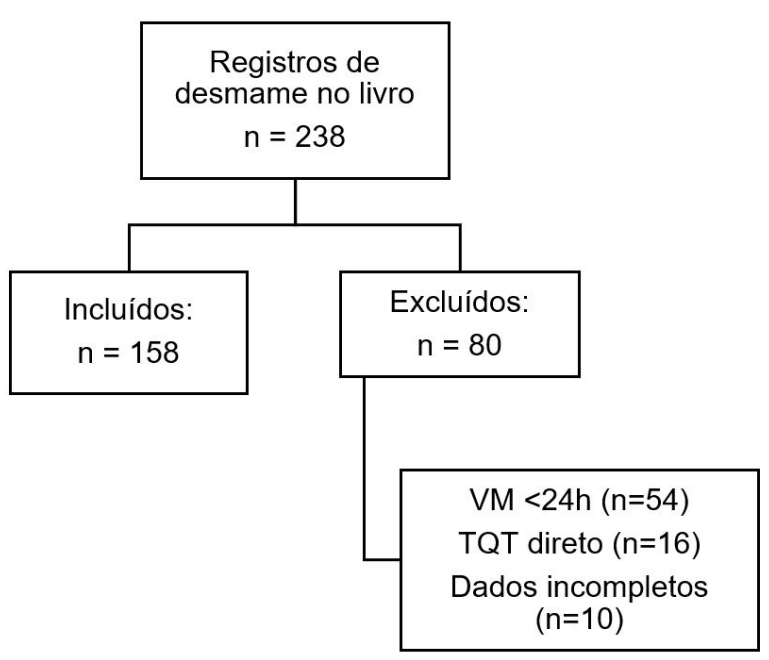

Figura 2. Fluxograma de seleção da amostra.

TQT: traqueostomia; VM: ventilação mecânica; n: número amostral. normalidade dos dados por meio do teste de KolmogorovSmirnov. Foi realizada análise estatística inferencial por meio do teste t não pareado de Student ou teste de Mann-Whitney para comparação das variáveis contínuas e teste exato de Fisher para comparação das variáveis categóricas. Para isto foi utilizado o programa Statistical Package for Social Sciences (SPSS), foram considerados estatisticamente significativos valores de $p \leq 0,05$.

\section{RESULTADOS}

De acordo com os dados coletados, foram identificados 238 pacientes submetidos a desmame da VM na UTI neurocirúrgica, destes, foram incluídos 158 pacientes que foram extubados (Figura 2).

As características sociodemográficas demonstraram prevalência do sexo masculino em ambos os grupos sucesso e falha no desmame ventilatório $(67 \%$ e $52,5 \%$ respectivamente), além disso, o grupo falha apresentou escore SAPS III significativamente maior $(p=0,003)$ que $o$ grupo sucesso (Tabela 1).

Entre os diagnósticos de admissão mais prevalentes, verificou-se o TCE (18\%) no grupo sucesso e a ressecção de tumor cerebral $(27,5 \%)$ no grupo falha (Tabela 2 ). Além disso, verificou-se outros diagnósticos nos grupos sucesso (21\%) e falha (15\%), os quais incluíram artrodeses de coluna vertebral, choques sépticos de origem meningite, hidrocefalias.

Entre as comorbidades, verificou-se a hipertensão arterial sistêmica (HAS) em ambos os grupos sucesso (36\%)

Tabela 1. Características dos pacientes que evoluíram com sucesso ou falha no desmame da ventilação mecânica.

\begin{tabular}{cccc}
\hline Variável & Grupo Sucesso & Grupo Falha & p-valor \\
\hline Amostra & $\mathrm{n}=118$ & $\mathrm{n}=40$ & - \\
Sexo masculino, N (\%) & $79(67 \%)$ & $21(52,5 \%)$ & $0,12^{\dagger}$ \\
Idade (anos) & $50,5 \pm 16,9$ & $51,1 \pm 12,9$ & $0,84^{*}$ \\
SAPS III & $54,3 \pm 17,4$ & $68,8 \pm 14,6$ & $\mathbf{0 , 0 0 3}^{*}$
\end{tabular}

teste exato de Fisher. *teste $t$ não pareado. p-valor: teste estatístico utilizado para avaliar se há diferenças entre os grupos. n: número amostral; M: masculino. F: feminino.

Tabela 2. Diagnósticos de admissão mais prevalentes nos pacientes que evoluíram com sucesso ou falha no desmame da ventilação mecânica.

\begin{tabular}{|c|c|c|c|c|}
\hline \multirow{2}{*}{ Diagnóstico } & \multicolumn{2}{|c|}{ Grupo Sucesso } & \multicolumn{2}{|c|}{ Grupo Falha } \\
\hline & $\mathbf{n}$ & $\%$ & $\mathbf{n}$ & $\%$ \\
\hline AVC & 15 & 13 & 8 & 20 \\
\hline HSA aneurismática & 17 & 14,5 & 7 & 17,5 \\
\hline Ressecção de tumor cerebral & 16 & 13,5 & 11 & 27,5 \\
\hline Politrauma & 15 & 13 & 3 & 7,5 \\
\hline TCE & 21 & 18 & 5 & 12,5 \\
\hline Drenagem de hematoma cerebral & 9 & 8 & - & - \\
\hline Outros & 25 & 21 & 6 & 15 \\
\hline
\end{tabular}

AVC: acidente vascular cerebral; HSA: hemorragia subaracnóidea; TCE: traumatismo cranioencefálico. 
e falha (35\%) seguidas de etilismo e tabagismo, como as mais prevalentes (Tabela 3).

Entre as variáveis de desfecho verificou-se que o grupo falha permaneceu tempo significativamente maior $(p=0,05)$ de dias em VM e de internação $(p=0,003)$ em dias na UTI em comparação ao grupo sucesso, também se observou que o grupo sucesso apresentou percentual de alta significativamente maior que o grupo falha ( $90 \%$ versus $67,5 \% p=0,002$ ) do desmame ventilatório (Tabela 4).

\section{DISCUSSÃO}

No presente estudo foram incluídos 158 pacientes, $75 \%$ no grupo sucesso e $25 \%$ no grupo falha do desmame ventilatório, com predomínio do sexo masculino em ambos os grupos. O diagnóstico mais prevalente foi o traumatismo cranioencefálico (TCE) no grupo sucesso e a ressecção de tumor cerebral no grupo falha. O grupo falha apresentou escore SAPS III significativamente maior e permaneceu mediana de tempo em dias significativamente maior em VM e em internação na UTI que o grupo sucesso. Além disso, o grupo sucesso do desmame ventilatório apresentou o desfecho alta da UTI significativamente maior que o grupo falha.

Um estudo recente 6 avaliou o pico de fluxo expiratório como preditor de extubação em 135 pacientes neurológicos e constatou predomínio de pacientes do sexo masculino $(71,1 \%)$ e média de idade de $47,8 \pm 17$ anos. Este achado foi semelhante ao encontrado em nosso estudo, no qual houve prevalência do sexo masculino em ambos os grupos sucesso e falha no desmame ventilatório e a média de idade por volta dos 50 anos. Epidemiologicamente, a prevalência do sexo masculino está associada ao estilo de vida e maior exposição a fatores de risco para TCE, diagnóstico mais prevalente em nosso estudo ${ }^{6}$.

Lucena et al. ${ }^{18}$ identificaram os fatores de risco associados ao escore SAPS III para melhorar a predição de mortalidade em idosos internados em UTI. Os autores encontraram média geral de 43,6 para o escore SAPS III, sendo 38,7 entre os pacientes que receberam alta da UTI e 55 entre os que evoluíram a óbito. Esses resultados são contraditórios aos encontrados no presente estudo que, as médias do escore SAPS III foram 54 e 68 nos grupos sucesso e falha do desmame respectivamente. Essa diferença é explicada pelo fato de que no estudo avaliado a média de idade foi de 71,5 anos enquanto no presente estudo a média de idade é por volta de 50 anos para ambos os grupos sucesso e falha; um valor significativamente maior considerando que a idade é uma variável para determinação do valor do índice preditivo de mortalidade, visto que; segundo Stein et al. ${ }^{19}$ o envelhecimento, diminui as reservas funcionais, com isso, há declínio nas funções cardiovascular, pulmonar e renal, bem como diminuição de força, massa muscular e perda de memória. Essas modificações repercutem consideravelmente dentro da UTI, pois a idade do paciente por si só, é um fator que dificulta no manejo hemodinâmico e ventilatório.

Segundo dados da literatura, os diagnósticos clínicos mais prevalentes em pacientes com distúrbios neurológicos

Tabela 3. Comorbidades mais prevalentes nos pacientes que evoluíram com sucesso ou falha no desmame da ventilação mecânica.

\begin{tabular}{|c|c|c|c|c|}
\hline \multirow{2}{*}{ Comorbidade } & \multicolumn{2}{|c|}{ Grupo Sucesso } & \multicolumn{2}{|c|}{ Grupo Falha } \\
\hline & $\mathbf{n}$ & $\%$ & $\mathbf{n}$ & $\%$ \\
\hline Hipertensão arterial sistêmica & 43 & 36 & 14 & 35 \\
\hline Diabetes mellitus & 13 & 11 & 4 & 10 \\
\hline Obesidade & 3 & 2,5 & 2 & 5 \\
\hline Dislipidemias & 6 & 5 & - & - \\
\hline Doença renal crônica & 3 & 2,5 & 2 & 5 \\
\hline Etilismo & 25 & 21 & 10 & 25 \\
\hline Tabagismo & 25 & 21 & 12 & 30 \\
\hline
\end{tabular}

n: número amostral.

Tabela 4. Comparação das variáveis de desfecho dos pacientes que evoluíram com sucesso ou falha no desmame da ventilação mecânica.

\begin{tabular}{cccc}
\hline Variável & Grupo Sucesso & Grupo Falha & p-valor \\
\hline Tempo de VM (dias) & $2[1-12]$ & $3,5[1-14]$ & $\mathbf{0 , 0 5 ^ { * }}$ \\
Internação na UTI (dias) & $9[2-49]$ & $12,5[5-35]$ & $\mathbf{0 , 0 0 3 *}^{*}$ \\
Desfecho na UTI & Alta=106 (90\%) & Alta $=27(67,5 \%)$ & $\mathbf{0 , 0 0 2}^{\dagger}$
\end{tabular}

*teste de Mann-Whitney; ${ }^{\dagger}$ teste exato de Fisher. p-valor: teste estatístico utilizado para avaliar se há diferenças entre os grupos. 
são o TCE (47\%), seguido por hemorragia subaracnóidea $(35,6 \%)$, hemorragia intracraniana $(11,4 \%)$, e tumores cerebrais $(6,1 \%)^{20}$. Esses resultados se assemelham aos achados epidemiológicos do presente estudo. Tais resultados podem ser explicados por questões de gênero e hábitos de vida.

Silva et al. ${ }^{21}$ descreveram e analisaram a relação entre presença de comorbidades e desenvolvimento de PAV em pacientes internados em UTI. Os autores identificaram que as comorbidades mais prevalentes foram as afecções cardiovasculares $(35,7 \%)$ seguidas do tabagismo $(27,1 \%)$. Esses resultados corroboram os encontrados no presente estudo, a HAS seguida de etilismo e tabagismo foram as mais prevalentes. O etilismo e o tabagismo são os principais fatores de risco para HAS, principal fator causal de um AVE, o que demonstra a importância de medidas de prevenção e conscientização para a população em geral ${ }^{22}$.

Verificou-se entre as variáveis de desfecho que o grupo falha do desmame ventilatório permaneceu maior tempo em VM e internação, resultando assim em menor índice de alta e maior mortalidade. Condizendo com o que foi visto na literatura ${ }^{23,24}$. A VM prolongada está relacionada ao desenvolvimento de patologias como PAV, disfunção diafragmática induzida pela ventilação, lesão pulmonar induzida pela ventilação e polineuropatia, além de aumentar a mortalidade ${ }^{14}$.

A presente pesquisa apresentou algumas limitações em relação ao fato de ser do tipo retrospectivo e realizado em uma única UTI, sendo, portanto, representativos da prática clínica desta unidade. Excluímos os pacientes que necessitaram de VM por tempo inferior a $24 \mathrm{~h}$ e pacientes que evoluíram para traqueostomia direto. Outra limitação refere-se ao fato de não ter sido estratificado, nem realizado comparações estatísticas dos desfechos de quais e quantos TREs os pacientes dos dois grupos foram submetidos, pois sabe-se que o TRE realizado com tubo T se aproxima muito do trabalho respiratório do paciente em ventilação espontânea, porém, isto se deu aos dados incompletos no livro de registro de desmame ventilatório do Serviço de Fisioterapia, o que implica um possível viés do estudo.

A revisão do livro de desmame ventilatório do Serviço de Fisioterapia revelou que os dados não foram registrados de forma integral durante os anos, acarretando perda de informações importantes para o delineamento do perfil clínico dos pacientes admitidos e submetidos ao processo de desmame. Essas informações são importantes para o fisioterapeuta intensivista para nortear estratégias facilitadoras do desmame ventilatório em grupos específicos de pacientes, como os neurológicos.

\section{CONCLUSÃO}

No presente estudo os pacientes do grupo falha do desmame apresentaram maior para mortalidade na UTI, maior tempo, em dias, de ventilação mecânica invasiva e de internação na UTI neurocirúrgica, menor taxa de alta e maior taxa de óbitos na comparação com o grupo sucesso do desmame ventilatório.

\section{FONTE DE FINANCIAMENTO}

Nada a declarar.

\section{CONFLITO DE INTERESSES}

Nada a declarar.

\section{REFERÊNCIAS}

1. Alves LCB, Thommen LP, Gomes CM, Oliveira VRC. Conhecimento de acadêmicos da saúde sobre a atuação do fisioterapeuta em unidades de terapia intensiva. Rev Bras Mil Cien. 2019;5(13):34-9. http://dx.doi.org/10.36414/rbmc. v5i13.17.

2. Dornelles C, Oliveira GBD, Schwonke CRG, Silva JRDS. Experiências de doentes críticos com a ventilação mecânica invasiva. Esc Anna Nery. 2012;16(4):796-801. http://dx.doi. org/10.1590/S1414-81452012000400022.

3. Santos CD, Nascimento ERPD, Hermida PMV, Silva TG, Galetto SGDS, Silva NJCD, et al. Boas práticas de enfermagem a pacientes em ventilação mecânica invasiva na emergência hospitalar. Esc Anna Nery. 2020;24(2):1-7. http://dx.doi. org/10.1590/2177-9465-ean-2019-0300.

4. da Cruz J, Martins M. Pneumonia associada à ventilação mecânica invasiva: cuidados de enfermagem. Rev Enf Referen. 2019;4(20):87-96. http://dx.doi.org/10.12707/ RIV18035.

5. Pinheiro RA, Christofoletti G. Fisioterapia motora em pacientes internados na unidade de terapia intensiva: uma revisão sistemática. Rev Bras Ter Intensiva. 2012;24(2):18896. http://dx.doi.org/10.1590/S0103-507X2012000200016. PMid:23917769.

6. Kutchak FM, Debesaitys AM, Rieder MM, Meneguzzi C, Skueresky AS, Forgiarini Junior LA, et al. Reflex cough PEF as a predictor of successful extubation in neurological patients. J Bras Pneumol. 2015 Jul-Ago;41(4):358-64. http://dx.doi. org/10.1590/S1806-37132015000004453. PMid:26398756.

7. Oliveira FIL, Souza KZFN, Roberto SBA, Alencar CH, Ferreira FA. Fatores que contribuem para o insucesso do desmame ventilatório no paciente grave. Rev Fama Cien Saúde. 2015;1(1):13-9.

8. Goldwasser R, Farias A, Freitas EE, Saddy F, Amado V, Okamoto V. III Consenso Brasileiro de Ventilação Mecânica: Desmame e interrupção da ventilação mecânica. Sociedade Brasileira de Pneumologia e Tisiologia. J Bras Pneumol. 2007;33(2):128-36.

9. Associação de Medicina Intensiva Brasileira. Sociedade Brasileira de Pneumologia e Tisiologia. Diretrizes Brasileiras de Ventilação Mecânica. São Paulo: Associação de Medicina Intensiva Brasileira; 2013.

10. Muzaffar SN, Gurjar M, Baronia AK, Azim A, Mishra P, Poddar $B$, et al. Predictors and pattern of weaning and long-term outcome of patients with prolonged mechanical ventilation at an acute intensive care unit in North Índia. Rev Bras Ter Intensiva. 2017;29(1):23-33. http://dx.doi.org/10.5935/0103507X.20170005. PMid:28444069. 
11. Loss SH, de Oliveira RP, Maccari JG, Savi A, Boniatti MM, Hetzel MP, et al. The reality of patients requiring prolonged mechanical ventilation: a multicenter study. Rev Bras Ter Intensiva. 2015;27(1):26-35. http://dx.doi.org/10.5935/0103507X.20150006. PMid:25909310.

12. Sun Y, Li S, Wang S, Li C, Li G, Xu J, et al. Predictors of 1-year mortality in patients on prolonged mechanical ventilation after surgery in intensive care unit: a multicenter, retrospective cohort study. BMC Anesthesiol. 2020;20(1):44. http://dx.doi. org/10.1186/s12871-020-0942-0. PMid:32085744.

13. Jesus FS, Paim DM, Brito JO, Barros IA, Nogueira TB, Martinez $B P$, et al. Declínio da mobilidade dos pacientes internados em unidade de terapia intensiva. Rev Bras Ter Intensiva. 2016;28(2):114-9. PMid:27410406.

14. Marinho RI, Silva RAD, Farias CAC, Silva JDS, Macêdo LCB, Scön CF, et al. Desmame da ventilação mecânica de pacientes críticos adultos: revisão sobre a utilização de protocolos. Cad Educ Saúde Fisioter. 2018;5(10):53-63.

15. Gutiérrez EP, Díaz JSS, Hernandez RF, Rodriguez EAM, Peniche KG, Gutiérrez SPD, et al. Los predictores en el retiro de la ventilación mecánica resultan suficientes para el paciente neurocrítico? Med Int México. 2017;33(5):675-91.

16. Balaji B, Rao AB, Kumar VS. Sammaiah. Performance of simplified acute physiology score 3 admission score as a predictor of ICU mortality in a tertiary care hospital of rural Telangana, India. Int J Adv Med. 2017;3(3):716-20.

17. Moralez GM, Rabello LSCF, Lisboa TC, Lima MFA, Hatum RM, De Marco FVC, et al. External validation of SAPS 3 and MPM0-III scores in 48,816 patients from 72 Brazilian ICUs. Ann Intensive Care. 2017;7(1):53. http://dx.doi.org/10.1186/ s13613-017-0276-3. PMid:28523584.

18. Lucena MVF, Veloso MAOS, Santos-Xavier VR, Ferreira IB, Moraes FC, Lucena RA, Lucena LA, Markman-Filho
B, Lima SG. Mortality prognostic factors associated with SAPS 3 and APACHE II in elderly patients admitted in intensive care unit. Medicina (Ribeirão Preto, Online). 2019;52(4):277-86. http://dx.doi.org/10.11606/issn.21767262.v52i4p277-285.

19. Stein FC, Barros RK, Feitosa FS, Toledo DO, Silva JM Jr, Ísola AM, et al. Fatores prognósticos em pacientes idosos admitidos em unidade de terapia intensiva. Rev Bras Ter Intensiva. 2009;21(3):255-61. http://dx.doi.org/10.1590/ S0103-507X2009000300004. PMid:25303546.

20. Asehnoune K, Seguin P, Lasocki S, Roquilly A, Delater A, Gros A, et al. Extubation success prediction in a multicentric cohort of patients with severe brain injury. Anesthesiol. 2017;127(2):338-46. http://dx.doi.org/10.1097/ ALN.0000000000001725. PMid:28640020.

21. Silva RM, Silvestre MO, Zocche TL, Sakae TM. Pneumonia associada à ventilação mecânica: fatores de risco. Rev Bras Clin Med. 2011;9(1):5-10.

22. Vieira AM, Parente EA, Oliveira LS, Queiroz AL, Bezerra ISAM, Rocha HAL. Características de óbitos dos pacientes internados em uma unidade de terapia intensiva de hospital terciário. J Health \& Biol Sciences. 2018;7(1):26-31. http:// dx.doi.org/10.12662/2317-3076jhbs.v7i1.1999.p26-31.2019.

23. Rishi MA, Kashyap R, Wilson G, Schenck L, Hocker S. Association of extubation failure and functional outcomes in patients with acute neurologic illness. Neurocrit Care. 2016;24(2):217-25. http://dx.doi.org/10.1007/s12028-0150156-3. PMid:26215402.

24. Cheng AC, Cheng KC, Chen CM, Hsing SC, Sung MY. The outcome and predictors of failed extubation in intensive care patients - the elderly is an important predictor. Int J Gerontol. 2011;5(4):206-11. http://dx.doi.org/10.1016/j. ijge.2011.09.021. 